\title{
Bacterial isolates and drug susceptibility patterns of urinary tract infection among pregnant women at Muhimbili National Hospital in Tanzania
}

\author{
SABRINA J. MOYO ${ }^{3 * 1}$, SAID ABOUD ${ }^{1}$, MABULA KASUBI ${ }^{2}$ and SAMUEL Y. MASELLE ${ }^{1}$ \\ ${ }^{I}$ Department of Microbiology and Immunology, Muhimbili University of Health and Allied Sciences, Dar es \\ Salaam, Tanzania; \\ ${ }^{2}$ Department of Laboratory Services, Microbiology Section, Central Pathology Laboratory, Muhimbili \\ National Hospital, Dar es Salaam, Tanzania
}

\begin{abstract}
Urinary tract infection (UTI) during pregnancy may cause complications such as pyelonephritis, hypertensive disease of pregnancy, anaemia, chronic renal failure, premature delivery and foetal mortality. This study aimed to identify the etiologic agents of UTI and to determine the patterns of antimicrobial drug susceptibility among pregnant women at Muhimbili National Hospital in Tanzania. Retrospective analysis of 200 mid-stream urine specimens processed for culture and antimicrobial drug susceptibility testing between January 2007 and December 2009 was carried out. Significant bacteriuria (> 10 colony forming units/mL of urine) was found in 42/200 (21\%) specimens. Of the 42 isolates, the most commonly isolated bacteria were Escherichia coli 14 (33.3\%), Klebsiella spp 9 (21.4\%) coagulase negative Staphylococcus 7 (16.7\%), Staphylococcus aureus 6 (14.3\%), Proteus species 3 (7.1\%) and Enterococcus species 3 (7.1\%). Low rate to moderately high rate of antimicrobial drug resistance was observed against first line drugs namely, nitrofurantoin $18.7 \%(n=16)$, co-trimoxazole $38.5 \%(n=13)$ and ampicillin $57.7 \%(n=26)$. Relatively low rate of resistance was seen against second line drugs: ciprofloxacin $13.6 \%(n=22)$ and amikacin $5 \%(n=20)$. High rate of resistance was observed in third generation cephalosporin cefotaxime $31.2 \%(n=16)$. Of the Gram-positive organisms tested against vancomicin and methicilin, resistance was found in $25 \%(n=13)$ and $25 \%(n=4)$, respectively. In conclusion, E coli was found to be the common cause of UTI among the pregnant women. Low to moderately high level of resistance was found in first line drugs while high level of resistance was found in third generation cephalosporin. It is recommended to monitor the levels of resistance for nitrofurantoin, fluoroquinolone and cefotaxime and to screen for Extended Spectrum Beta Lactamase production among cefotaxime resistant $E$. coli and Klebsiella spp.
\end{abstract}

Key words: Urinary tract infection, pregnant women, antimicrobial drug resistance, Tanzania

\section{Introduction}

Urinary tract infection (UTI) is a common health problem among pregnant women (Mittal \& Wing, 2005; Saidi et al., 2005). This usually begins in week 6 and peaks during weeks 22 to 24 of pregnancy due to a number of factors including ureteral dilatation, increased bladder volume and decreased bladder tone, along with decreased ureteral tone which contributes to increased urinary stasis and ureterovesical reflux (Chaliha et al., 2002). Up to $70 \%$ of pregnant women develop glycosuria, which encourages bacterial growth in the urine (Al-Issa, 2009).

UTI may manifest as asymptomatic

3 " Correspondence to: Dr. Sabrina J. Moyo; E-mail: sabmoyo26@yahoo.com/_smoyo@muhas.ac.tz bacteriuria (ASB) or symptomatic bacteriuria (SB). The prevalence of asymptomatic UTI has been previously reported to be $2 \%$ to $13 \%$ in pregnant women (Delzell et al., 2000; Christensen, 2000; Kutlay et al., 2003; McIsaac ., 2005; Masinde et al., 2009) compared with that of symptomatic UTI which occurs in 1-18\% during pregnancy (Dwyer \& O'Reilly, 2002; Masinde et al., 2009).

UTI during pregnancy may cause complications such as pyelonephritis, hypertensive disease of pregnancy, anaemia, chronic renal failure, premature delivery and foetal mortality (Delzell et al., 2000; Foxman, 2002). The incidence of these complications can be decreased by treating promptly ASB and SB during pregnancy (Delzell et al., 2000). Due to the potential adverse sequelae of UTI in pregnancy, most clinics perform routine 
urinalysis of midstream urine specimen during one or more antenatal clinic (ANC) visits (Smaill, 2007). However, culture and antimicrobial drug susceptibility testing are needed for surveillance purposes to guide the clinicians on the proper management and prevent empirical treatment of pregnant women with ASB and SB.

A limited spectrum of organisms cause UTI and these include Escherichia coli, which accounts for the majority of uncomplicated UTI isolates (Gupta et al., 2001; Nicolle, 2001; Masinde et al., 2009). Others are Staphylococcus saprophyticus, Klebsiella spp, Proteus spp, Enterococcus spp, and Enterobacter spp (Gupta et al., 2001; Masinde et al., 2009). Data on the current distribution and antimicrobial drug susceptibility patterns among urinary bacterial isolates from pregnant women in Tanzania is limited. This study aimed to identify the etiologic agents of UTI and to determine the patterns of antimicrobial drug susceptibility among pregnant women at Muhimbili National Hospital (MNH), Dar es Salaam, Tanzania.

\section{Material and methods}

\section{Study area}

A retrospective review and analysis of 3-year laboratory records (2007-2009) on urine culture specimens from pregnant women attending ANC at Muhimbili National Hospital in Tanzania was carried out. Data were extracted from laboratory registers in the Microbiology Section at Central Pathology Laboratory (CPL) of the Hospital. Information on the type of bacterial isolates from the urine specimens and antibiotic susceptibility patterns were recorded.

\section{Bacterial isolation and antimicrobial susceptibility testing}

Urine samples received at the Central Pathology
Laboratory (CPL) were platted on cysteine lactose electrolytes deficient (CLED) agar medium incubated at $37^{\circ} \mathrm{C}$ for 24 hours. Growth of one type of organism of $>10^{5}$ colony forming units was considered as significant bacteriuria. Identification of isolates was done by observing colonial morphology on CLED medium. Colonies were further identified using standard biochemical tests as described previously (McCartney et al., 1989).

Kirby-Bauer disk diffusion test was used to perform antimicrobial susceptibility testing for all the isolates as recommended by Clinical and Laboratory Standard Institute (CLSI 2006). Antibiotic disks (Oxoid) were applied to each plate. Inhibition zone sizes were measured and interpreted according to CLSI guidelines. Antimicrobial drug susceptibility testing for cotrimoxazole, ampicillin, ciprofloxacin, amikacin, nitrofurantoin, methicilin, vancomicin and cefotaxime was done on all bacteria isolated.

\section{Results}

During the period of 2007-2009, a total of 200 urine specimens was received from ANC and processed. Significant bacteriuria (cultures with $>10^{5}$ colony forming units (cfu) of bacteria/mL of urine) was found in $42 / 200(21 \%$; range $=18.7-23.2 \%)$ of the urine specimens. Mixed bacterial growths were observed in 14 specimens (Table 1). Of all the bacteria isolated, Gram-negative bacteria were more prevalent $(61.9 \%)$ than Gram-positive bacteria which constituted $38.1 \%$. The most commonly isolated bacteria were Escherichia coli 14 (33.3\%), Klebsiella species $9(21.4 \%)$, coagulase negative Staphylococcus 7 (16.7\%), S. aureus 6 (14.3\%), Proteus species $3(7.1 \%)$ and Enterococcus species $3(7.1 \%)$.

Table 1: Distribution of bacteria isolated from pregnant women presenting with symptoms of UTI at MNH, Dar es Salaam, Tanzania, from 2007 to 2009.

\begin{tabular}{lllll}
\hline Organism isolated & $\mathbf{2 0 0 7}$ & $\mathbf{2 0 0 8}$ & $\mathbf{2 0 0 9}$ & Total (\%) \\
\hline Escherichia coli & 4 & 7 & 3 & $14(33.3)$ \\
Klebsiella species & 2 & 3 & 4 & $9(21.4)$ \\
Proteus species & 1 & 1 & 1 & $3(7.1)$ \\
S. aureus & 4 & 0 & 2 & $6(14.3)$ \\
Coagulase negative staphylococcus & 3 & 2 & 2 & $7(16.7)$ \\
Enterococcus species & 3 & 0 & 0 & $3(7.1)$ \\
Total number of urine specimen received & 91 & 56 & 53 & 200 \\
Total positive urine culture & 17 & 13 & 12 & 42 \\
\hline
\end{tabular}


Overall bacterial sensitivity to first line antibiotics for UTI was: nitrofurantoin $81.3 \%(\mathrm{n}=16)$, ampicillin $42.3 \%(n=26)$ and co-trimoxazole $61.5 \%(n=13)$. Sensitivity to second line drugs was: ciprofloxacin $86.4 \%(n=22)$ and amikacin $95 \%(n=20)$. Gram negative bacteria showed a $31.2 \%(\mathrm{n}=16)$ resistance to third generation cephalosporin cefotaxime (another second line antibiotic for UTI). Of the Gram-positive organisms tested against vancomicin and methicillin, antibiotic resistance was found in $25 \%(\mathrm{n}=13)$ and $25 \%(\mathrm{n}=4)$, respectively of the isolates (Table 2).

Table 2: Patterns of antimicrobial sensitivity results for the bacterial isolated from pregnant women presenting with UTI at MNH, Dare s Salaam, Tanzania, 2007-2009.

\begin{tabular}{lll}
\hline Antimicrobial agents (number of isolates tested, $\mathbf{n})$ & Sensitive isolates $(\mathbf{S})$ & Resistant isolates $(\mathbf{R})$ \\
\hline Sensitivity patterns for first line antimicrobial agents & & \\
Ampicillin $(\mathrm{n}=26) *$ & $11(42.3 \%)$ & $15(57.7 \%)$ \\
Cotrimoxazole $(\mathrm{n}=13) *$ & $8(61.5 \%)$ & $5(38.5 \%)$ \\
Nitrofurantoin $(\mathrm{n}=16) * *$ & $13(81.3 \%)$ & $3(18.7 \%)$ \\
Sensitivity patterns for second line antimicrobial agents & & $1(5 \%)$ \\
Amikacin $(\mathrm{n}=20) *$ & $19(95 \%)$ & $3(13.6 \%)$ \\
Ciprofloxacin $(\mathrm{n}=22) *$ & $19(86.4 \%)$ & $5(31.2 \%)$ \\
Cefotaxime $(\mathrm{n}=16) * *$ & $11(68.8 \%)$ & \\
Sensitivity pattern for gram positive cocci & & $1(25 \%)$ \\
Methicilin $(\mathrm{n}=4) * * *$ & $3(75 \%)$ & $1(25 \%)$ \\
Vancomicin $(\mathrm{n}=13) * * *$ & $12(95 \%)$ & \\
\hline
\end{tabular}

* tested against gram positive and gram negative bacteria; ** tested against gram negative only; *** tested against gram positive only; ****tested against Staphylococcus spp only

\section{Discussion}

Proper investigation and prompt treatment are needed to prevent serious life threatening condition and morbidity due to UTI that can occur in pregnant women. Our study findings showed that only 200 $(0.27 \%)$ urine specimens out of 73,894 antenatal clinic attendees were tested for culture and antibiotic susceptibility patterns during the last three years. The small number of specimens may be explained by the fact that only those suspected to have UTI were investigated. We recommend screening of all pregnant women for significant bacteriuria because ASB can be present in $2 \%$ to $13 \%$ of pregnant women (Delzell et al., 2000; Kutlay et al. 2003; McIsaac et al., 2005; Masinde et al., 2009). Moreover, previous clinical trials have shown that antibiotic treatment significantly reduces the risk of complications associated with UTI in pregnancy. Furthermore, screening for and treatment of asymptomatic bacteriuria during pregnancy has become a standard of care in other countries (Smaill, 2007).

Of the urine specimens from pregnant women suspected to have UTI, about one-fifth was positive for bacterial cultures with significant bacteriuria. This is similar to the findings reported previously in Ethiopia (Assefa et al., 2008) but slightly higher than that reported by Olsen et al. (2000) and Masinde et al. (2009) in Arusha and Mwanza, Tanzania. Treatment of both SB and
ASB in pregnancy can prevent occurrence of adverse outcomes for mother such as septicaemia, pyelonephritis, hypertension, pre-eclampsia and premature labour as well as adverse outcome for the unborn child (Raz, 2003).

In this study, Gram negative bacteria were more prevalent (61.9\%) than Gram positive bacteria $(38.1 \%)$. Similar findings have been reported in rural Tanzania (Blomberg et al., 2005) as well as elsewhere (Gebre-Selassie, 1998; Delzell \& Lefevre, 2000; Nicolle, 2001; Schnarr \& Smaill , 2008). E. coli was major pathogen isolated from the urine cultures and accounted for one-third of the positive cultures with significant bacteriuria. $E$. coli is considered uropathogenic due to a number of virulence factors specific for colonisation and invasion of the urinary epithelium, such as the P-fimbria and S-fimbria adhesions (Sheffield \& Cunningham, 2005). Klebsiella species and coagulase negative Staphylococci were the second and third most common bacteria isolated.

Although the spectrum of agents causing UTI in pregnant women is relatively constant, their antibiotic susceptibility patterns are different in different geographical locations. The current study showed high level of resistance to first line antimicrobial drugs such as cotrimoxazole. These findings concur with findings from previous studies (Gupta et al., 2001; Arredondo-García et al. 2007; Arredondo-García \& Amábile-Cuevas, 
2008). Most antimicrobials used to treat UTI including cotrimoxazole can achieve high urinary concentrations. This could imply that urinary isolates which were moderately resistant could be effectively treated with normal doses of antibiotics. However, some previous studies have reported that clinical cure rates may be lower among outpatient women with uncomplicated UTI treated with cotrimoxazole when the infecting pathogen is resistant to cotrimoxazole (Masterton \& Bochsler, 1995; Talan et al., 2000, Raz et al., 2002). Low level of resistance was found against nitrofurantoin, and this might be due to its narrow range of clinical indications, which results in less usage. Considering the in vitro activity of nitrofurantoin and availability as an oral drug, nitrofurantoin should be considered as one of the drugs of choice against UTI in pregnant women where multi-resistant uropathogens are prevalent. However, the low resistance patterns of nitrofurantoin together with high resistance to cotrimoxazole observed in the current study call for the need to strengthen surveillance to identify changes in sensitivity pattern among urinary tract isolates.

The current study showed high level of cefotaxime resistance. Cefotaxime is second line antimicrobial agent in the third generation of cephalosporins. A possible explanation for the resistance found might be the presence of Extended Spectrum Beta-Lactamase (ESBL) in these strains. Noteworthy to mention that cefotaxime alone may not be enough as a screening drug for ESBL. However, this is an important finding, since it is likely to indicate that UTI in pregnant women at MNH may be present. Similar strains have been reported previously in septicaemic patients from the same hospital (Blomberg et al., 2005). It is therefore important that routine screening of ESBL in urinary isolates at $\mathrm{MNH}$ is carried out to prevent widespread of resistant isolates. The emergence of ESBLs in community-acquired infections has been reported recently (Arredondo-García \& AmábileCuevas, 2008). Resistance to ciprofloxacin although relatively low, could have been contributed by an increase in fluoroquinolone prescribing practices for uncomplicated UTI in recent years (Nicolle et al 2006).

This study is limited by the fact it included highly selected pregnant women with symptoms of UTI only and the duration of the study was only 3 -year period. Further studies are recommended to include both symptomatic and asymptomatic pregnant women with UTI over longer duration to establish a trend in antibiotic resistance pattern.
In conclusion, $E$ coli was found to be the common cause of UTI among the pregnant women. Low to moderately high level of resistance against first line drugs and high level of resistance against third generation cephalosporin was observed. It is recommended to monitor the levels of resistance for nitrofurantoin, fluoroquinolone and cefotaxime and screen for Extended Spectrum Beta Lactamase production among cefotaxime resistant E. coli and Klebsiella.

Received 17 March 2010

Revised 11 September 2010

Accepted 15 September 2010

\section{References}

Al-Issa, M. (2009) Urinary Tract Infection Among Pregnant Women in North Jordan. Middle East Journal of Family Medicine 7,

Arredondo-García, J.L. \& Amábile-Cuevas, C.F. (2008) High resistance prevalence towards ampicillin, co-trimoxazole and ciprofloxacin, among uropathogenic Escherichia coli isolates in Mexico City. Journal of Infection in Developing Countries 5, 350-353.

Arredondo-García, J.L., Soriano-Becerril, D., Solórzano-Santos, F., Arbo-Sosa, A., CoriaJiménez, R. \& Arzate-Barbosa, P. (2007) Resistance of uropathogenic bacteria to first-line antibiotics in Mexico City: a multicenter susceptibility analysis. Current Therapeutic Research 68, 120-126.

Assefa, A., Asrat, D., Woldeamanuel, Y., G/Hiwot, Y., Abdella, A. \& Melesse, T. (2008) Bacterial profile and drug susceptibility pattern of urinary tract infection in pregnant women at Tikur Anbessa Specialized Hospital Addis Ababa, Ethiopia. Ethiopian Medical Journal 3, 227-35.

Blomberg, B., Jureen, R., Manji, K.P., Tamim, B.S., Mwakagile, D.S.M., Urassa, W.K., Fataki, M., Msangi, V., Tellevik, M.G., Maselle, S.Y. \& Langeland, N. (2005) High rate of fatal cases of pediatric septicemia caused by Gram-negative bacteria with extendedspectrum beta-lactamases in Dar es Salaam, Tanzania. Journal of Clinical Microbiology 2, 745-749.

Chaliha, C. \& Stanton S.L. (2002) Urological problems in pregnancy. British Journal of Urology International 89, 469-476.

Christensen, B. (2000) Which antibiotics are 
appropriate for treating bacteriuria in pregnancy? Journal of Antimicrobial Chemotherapy 46, 29-34.

Clinical and Laboratory Standards Institute (2006) Performance Standards for Antimicrobial Susceptibility Testing; Sixteenth Informational Supplement. CLSI document M100-S16 [ISBN 1-56238-588-7]. Clinical and Laboratory Standards Institute.

Delzell, J.E. Jr \& Lefevre ML. (2000) Urinary tract infections during pregnancy. American Family Physician 61, 713-21.

Dwyer, P.L. \& O'Reilly M. (2002) Recurrent urinary tract infection in the female. Current Opinion in Obstetrics and Gynecology 14, 537-543.

Foxman, B. (2002) Epidemiology of urinary infections: incidence, morbidity, and economic costs. American Journal of Medicine 113A, 5S-113.

Gebre-Selassie, S. (1998)Asymptomatic bacteriuria in pregnancy: epidemiological, clinical and microbiological approach. Ethiopian Medical Journal 36, 185-92

Gupta, K., Hooten, T.M. \& Stamm, W.E. (2001) Increasing antimicrobial resistance and the management of uncomplicated communityacquired urinary tract infections. Annals of Internal Medicine 135, 41-50.

Kutlay, S., Kutlay, B., Karaahmetoglu, O., Ak, C. \& Erkaya, S. (2003) Prevalence, detection and treatment of asymptomatic bacteriuria in a Turkish obstetric population. Journal of Reproductive Medicine 48, 627-630.

Masinde, A., Gumodoka, B., Kilonzo, A. \& Mshana, S.E. (2009) Prevalence of urinary tract infection among pregnant women at Bugando Medical Centre, Mwanza, Tanzania. Tanzania Journal of Health Research 11, 154-159.

Masterton, R.G. \& Bochsler, J.A. (1995) Highdosage co-amoxiclav in a single dose versus 7 days of co-trimoxazole as treatment of uncomplicated lower urinary tract infection in women. Journal of Antimicrobial Chemotherapy 35, 129-137.

McCartney. M. (1989) Practical Medical Microbiology, Vol. 2, $13^{\text {th }}$ edn: Churchill Livingstone.

McIsaac, W., Carroll, J.C., Biringer, A., Bernstein P., Lyons E., Low D.E., Permaul J.A.. (2005) Screening for asymptomatic bacteriuria in pregnancy. Journal of Obstetrics and Gynaecology Canada 27, 20-24.
Mittal, P. \& Wing, D.A. (2005)Urinary tract infections in pregnancy. Clinics in Perinatology 32, 749-764.

Nicolle, L.E. (2001) Epidemiology of urinary tract infection. Infections in Medicine 18, 153162.

Nicolle, L., Anderson, P., Conly, J., Curtis Nickel, J., Senikas, \& Zhanel, G.G. (2006) Uncomplicated urinary tract infection in women Current practice and the effect of antibiotic resistance on empiric treatment. Canadian Family Physician 10,612-618.

Olsen, B.E., Hinderaker, S.G., Lie, R.T., Gasheka, P., Baerheim, A., Bergsjø, P. \& Kvåle, G. (2000) The diagnosis of urinary tract infections among pregnant women in rural Tanzania; prevalences and correspondence between different diagnostic methods. Acta Obstetricia et Gynecologica Scandinavica 9, 729-736.

Raz, R. (2003) Asymptomatic bacteriuria. Clinical significance and management. International Journal of Antimicrobial Agents 22, 45-7.

Raz, R., Chazan, B., Kennes, Y., Colodner, R., Rottensterich, E., Dan, M., Lavi, I., Stamm, W. \& the Israeli Urinary Tract Infection Group (2002) Empiric use of trimethoprimsulfamethoxazole (TMP-SMX) in the treatment of women with uncomplicated urinary tract infections, in a geographical area with a high prevalence of TMP-SMXresistant uropathogens. Clinical Infectious Diseases 34, 1165-1169.

Saidi, A., Delaporte, V. \& Lechevallier, E. (2005) Urological problems encountered during pregnancy. Progres en Urologie 15, 1-5.

Schnarr, J. \& Smaill F. (2008)Asymptomatic bacteriuria and symptomatic urinary tract infections in pregnancy. European Journal of Clinical Investigation 2, 50-57.

Sheffield, J.S. \& Cunningham, F.G. (2005) Urinary tract infection in women. Obstetrics \& Gynecology 106, 1085-1092.

Smaill, F. (2007) Asymptomatic bacteriuria in pregnancy. Best Practice \& Research 21, 439-450

Talan, D.A., Stamm, W.E., Hooton, T.M., Moran, G.J., Burke, T., Iravani, A., Reuning-Scherer, J. \& Church, D.A. (2000) Comparison of ciprofloxacin (7 days) and trimethoprimsulfamethoxazole (14 days) for acute uncomplicated pyelonephritis in women-a randomized trial. Journal of American Medical Association 283, 1583-1590. 